Hence $\lim _{n} c_{n}$ and $\lim _{n} b_{n}$ exist and are equal, i.e. $\lim _{n} \overline{\lim }_{m} a_{m n}$ exists. From the symmetry of the condition, we conclude that $\lim _{m}{\lim _{n}}_{n n}$ exists also. The identity of the two limits is then a consequence of the condition of our theorem and Statement A.

We note finally that the Cauchy condition for convergence of the double limit, $\lim _{m n} a_{m n}$, is the special case of our condition in which $m_{\epsilon n_{1}}$ and $n_{\epsilon m_{1}}$ are independent of $n_{1}$ and $m_{1}$ respectively, and can therefore be taken as $m_{e}$ and $n_{\epsilon}$, respectively.

UNIVERSITY OF MICHIGAN

\title{
ON BOUNDED REGULAR FRONTIERS IN THE PLANE*
}

\author{
BY W. A. WILSON
}

1. Introduction. The term regular frontier has been introduced by $\mathrm{P}$. Urysohn $\uparrow$ to designate a continuum which is the frontier of two or more components of its complement. Regular frontiers in the plane have been discussed by various authors. A. Rosenthal $\ddagger$ has shown that a continuum which is the union of two bounded continua that are irreducible between the same pair of points and have no other common points is a regular frontier. R. L. Moore§ has given necessary and sufficient conditions that a bounded continuum be a regular frontier whose complement has exactly two components. C. Kuratowski\| has given necessary conditions for a continuum to be a regular frontier which is the frontier of every component of its complement.

* Presented to the Society, October 29, 1927.

$\dagger \mathrm{P}$. Urysohn, Mémoire sur les multiplicités Cantoriennes, Fundamenta Mathematicae, vol. 7, p. 98.

$\ddagger$ A. Rosenthal, Teilung der Ebene durch Irreduzible Kontinua, Sitzungsberichte der Münchener Akademie, 1919.

$\S$ R. L. Moore, Concerning the common boundary of two domains, Fundamenta Mathematicae, vol. 6, pp. 203-213.

\| C. Kuratowski, Sur les coupures du plan, Fundamenta Mathematicae, vol. 6 , pp. 130-145. 
In the present article Rosenthal's theorem is generalized to cover the case of two continua having a finite or enumerably infinite set of closed sets in common and irreducible between each pair. With this result and a theorem by the author $\dagger$ given elsewhere, it is possible to formulate necessary and sufficient conditions for a bounded continuum to be a regular frontier. The principal theorems are to be found in $\S \S 5-7$.

2. Notation. Besides the ordinary notation of the aggregate theory the following special notation and terminology will be used.

The whole plane will be denoted by $Z$. If $A$ is a sub-set of $C$, the set of inner points of $A$ relative to $C$ will be denoted by $A .^{*}$ If $A$ and $B$ are two sets in the plane $Z$ without common points and $C$ separates $A$ from $B$ (i.e., $C \cdot A=C \cdot B=0$ and every continuum in the plane which contains points of both $A$ and $B$ also contains one or more points of $C$ ), we say that $C$ is an $S(A, B)$. If $F$ is a frontier set, a component of $Z-F$ which has the frontier $F$ will be called a principal component.

The statement " $C$ is a continuum irreducible between the sets $A$ and $B$ " will apply not only to the case that $A+B \subset C$, but also to the case that $C$ is irreducible between each point of $C \cdot A$ and each point of $C \cdot B$.

3. Some Auxiliary Theorems. Let $F=H+K$ be the union of two bounded continua and let $H \cdot K$ be the sum of a finite set of closed sets $\{\alpha\}$, such that both $H$ and $K$ are irreducible between each pair. The following properties of $F$ are either well known or so easily established that the proofs are omitted.

(a) If the number of sets $\{\alpha\}$ is greater than two, both $H$ and $K$ are indecomposable.

(b) If $C$ is a sub-continuum of $H$ or of $K, F-C$ is connected.

(c) $H^{*}=F-K$ and $K^{*}=F-H$ are connected; also $\bar{H}^{*}=H$ and $\bar{K}^{*}=K$.

$\dagger \mathrm{W}$. A. Wilson, On irreducible cuts of the plane between two points. (To appear soon in Annals of Mathematics.) 
If $F$ lies in a plane $Z$, certain properties expressing relations between $F$ and the rest of the plane can be proved without difficulty.

(d) If $H \cdot K$ has at least $n$ components, there are at least $n$ components of $Z-F$ which have frontier points on both $H^{*}$ and $K^{*}$. If $H \cdot K$ has an infinity of components, the components of $Z-F$ having frontier points on both $H^{*}$ and $K^{*}$ are enumerably infinite.

These statements are proved by adding to $H$ and $K$ those components of $Z-F$ which do not have frontier points on both $H^{*}$ and $K^{*}$ and applying a theorem of S. Straszewicz. $\dagger$

(e) There is a bounded continuum $P$ which is an irreducible $S\left(H^{*}, K^{*}\right)$ and contains $H \cdot K$.

To establish this we observe that, since $H^{*}$ and $K^{*}$ are connected, there is a bounded continum $Q$ which is an $S\left(H^{*}, K^{*}\right)$ by a theorem of Knaster and Kuratowski. $\ddagger$ But by a theorem of Mazurkiewicz, $\S Q$ contains a subcontinuum $P$ which is an irreducible $S\left(H^{*}, K^{*}\right)$. Moreover $P \supset H \cdot K$, since every point of $H \cdot K$ is a common limiting point of $H^{*}$ and $K^{*}$.

It may be added that every bounded irreducible $S\left(H^{*}, K^{*}\right)$ is a continuum containing $H \cdot K$.

4. Lemma. Let $F=H+K$ be the union of two bounded continua and let $H \cdot K$ be the sum of a finite set of closed sets $\{\alpha\}$ between each pair of which both $H$ and $K$ are irreducible. Let $R$ be a component of $Z-F$ such that $\bar{R}$ contains a bounded continuum containing points of two or more sets $\alpha$ but no other points of $F$. Then $F$ is the frontier of $R$.

Proof. The principles of inversion permit us to demonstrate the lemma on the assumption that $R$ is bounded. Let

†S. Straszewicz, Über die Zerschneidung der Ebene, Fundamenta Mathematicae, vol. 7, p. 174.

$¥$ B. Knaster and C. Kuratowski, Sur les ensembles connexes, Fundamenta Mathematicae, vol. 2, p. 236.

$\S \mathrm{S}$. Mazurkiewicz, Sur un ensemble $G$, etc., Fundamenta Mathematicae, vol. 1, p. 63. 
$C$ be the continuum referred to above. Then $R$ contains a closed polygon $J$ (whose interior is $I$ ) so large that $C-C \cdot I$ has no component containing points of more than one $\alpha$. This is easily seen since the number of sets $\alpha$ is finite. On the other hand, if $\alpha_{1}$ and $\alpha_{2}$ are two sets met by $C$, there are sub-continua $A_{1}$ and $A_{2}$ of $C-C \cdot I$ irreducible between $\alpha_{1}$ and $\alpha_{2}$, respectively, and $J$, and $A_{1} \cdot A_{2}=0$. Let $M$ be an arc of $J$ irreducible beween $A_{1}$ and $A_{2}$. Then $Q=A_{1}+M+A_{2}$ is irreducible between $\alpha_{1}$ and $\alpha_{2}$ and does not meet $F-\left(\alpha_{1}\right.$ $\left.+\alpha_{2}\right)$.

Since $Q$ and $H$ are both irreducible between $\alpha_{1}$ and $\alpha_{2}$ and have only points of these sets in common, while $Q$ is not the union of two indecomposable continua, it follows by an extension of Rosenthal's theorem $\dagger$ that $Z-(Q+H)$ has two principal components $S_{1}$ and $S_{2}$. Similarly, let $T_{1}$ and $T_{2}$ be the principal components of $Z-(Q+K)$.

As $K^{*}$ is connected, it lies in but one component of $Z-(Q+H)$; suppose that $K^{*} \cdot S_{1}=0$. Since $(F+Q) \cdot S_{1}=0$ and $S_{1}$ has frontier points on $M, R \cdot S_{1} \neq 0$ and $S_{1} \subset R$. Likewise either $T_{1}$ or $T_{2}$, say $T_{1}$, is a part of $R$. As $F=H+K$, every point of $F$ is a frontier point of either $S_{1}$ or $T_{1}$, and a fortiori of $R$.

5. Theorem. Let $F=H+K$ be the union of two bounded continua and let $H \cdot K$ be the sum of a finite number $n$ of closed sets $\{\alpha\}$ between each pair of which both $H$ and $K$ are irreducible. Then the number of principal components of $Z-F$ is at least $n$.

Proof. Let $R_{i}$ be any component of $Z-F$ having frontier points on both $H^{*}$ and $K^{*}$, let $P$ be a bounded irreducible $S\left(H^{*}, K^{*}\right)$, and let $P_{i}=P \cdot R_{i}$. Suppose that $\bar{P}_{i}$ has a subcontinuum containing points of more than one set $\alpha$ for only $k$ values of $i$, say $i=1,2, \cdots, k$, where $k<n$. We first show that $k \geqq 1$. For, if $\alpha^{\prime}$ denotes any $\alpha$ and $\alpha^{\prime \prime}$ the sum of the remaining sets $\alpha$, the continuum $P$ contains a connected

$\dagger \mathrm{W}$. A. Wilson, On the separation of the plane by irreducible continua, this Bulletin, vol. 33, pp. 733-744, §5. 
set having no points of $\alpha^{\prime}$ or $\alpha^{\prime \prime}$, but limiting points on both. This connected set must be a part of some $P_{i}$.

In some work done elsewhere $\nmid$ it was shown that there is a continuum $Q$, which is a bounded irreducible $S\left(H^{*}, K^{*}\right)$, constructed as follows: $Q$ is the union of $k$ arcs $\left\{A_{i}\right\}$, each $A_{i}$ lying in $R_{i}, i=1,2, \cdots, k$, and $k$ continua $\left\{B_{i}\right\}$, such that $B_{i} \cdot B_{i^{\prime}}=0$ if $i \neq i^{\prime}$, each $B_{i}$ is irreducible between an end of $A_{i}$ and one of $A_{i+1}(i+1=1$, if $i=k)$ and meets no other points of $\sum_{1}^{k} A_{i}$, and $H \cdot K \subset \sum_{1}^{k} B_{i} \subset P$. Moreover, in the demonstration referred to, the circle used may be replaced by a polygon so large that, if $I_{i}$ is its interior, no component of $\bar{P}_{i}-\bar{P}_{i} \cdot I_{i}$ has points on two sets $\alpha$. Consequently any connected sub-set of any $B_{i}$ having limiting points on two sets $\alpha$, but no points on $F$, must lie in some $R_{i}, i>k$.

Since $n>k$, some $B_{i}$ contains points of two or more sets $\alpha$. Hence for some $i>k$, there is a connected sub-set $j$ of $B_{i}$ having limiting points on two sets $\alpha$, but containing no points of $F$. Then $j$ lies in some $P_{i}$, where $i>k$ by the last part of the previous paragraph. Therefore $\bar{P}_{i}$ contains points of more than one set $\alpha$, contrary to the assumption at the beginning of the proof.

Therefore, for at least $n$ values of $i, \bar{P}_{i}$, and consequently $\bar{R}_{i}$, contains a continuum having points on more than one set $\alpha$. Then $\S 4$ shows that $F$ is the frontier of each such $R_{i}$. Hence the theorem is proved.

Corollary. Let $F=H+K$ be the union of two bounded continua and for every integer $n$ let $H \cdot K$ be the sum of $n$ closed sets such that both $H$ and $K$ are irreducible between each pair. Then the number of principal components of $Z-F$ is infinite.

† See reference to paper by the author under $\$ 1, \S \S 6$ and 7 . The hypothesis in these sections requires $R$ to be a principal component of $Z-F$, but the demonstration only requires that $R$ have frontier points on both $H^{*}$ and $K^{*}$.

$\ddagger$ Anna M. Mullikin, Certain theorems relating to plane connected sets, Transactions of this Society, vol. 24, Theorem 1. 
6. Theorem. For the bounded decomposable continuum $F$ to be the frontier of exactly $n$ components of its complement, it is necessary and sufficient that $F$ be the union of two continua $H$ and $K$ such that $H \cdot K$ is the sum of $n$, but of no finite number greater than $n$, closed sets between each pair of which both $H$ and $K$ are irreducible.

Proof. If $F$ is decomposable and $Z-F$ has $n$ principal components, it has been shown elsewhere that $F$ is the union of two continua $H$ and $K$ such that $H \cdot K$ is the sum of a finite number, greater than or equal to $n$, of closed sets between each pair of which both $H$ and $K$ are irreducible. On the other hand, if $H \cdot K$ can be expressed as the sum of $n$ closed sets between each pair of which both $H$ and $K$ are irreducible, there are at least $n$ principal components of $Z-F$ by $\$ 5$.

The combination of these statements gives the theorem.

Remarks. The decomposition of $H \cdot K$ into $n$ closed sets given above is unique. For, if there were two different decompositions into $n$ closed sets with the properties assigned, say $H \cdot K=\sum_{1}^{n} \alpha_{i}$ and $H \cdot K=\sum_{1}^{n} \beta_{i}$, some $\beta_{i}$, say $\beta_{1}$, would contain points of more than one set $\alpha_{i}$. Let $\beta_{1} \cdot \alpha_{1} \neq 0$ and let $\beta_{11}=\beta_{1} \cdot \alpha_{1}$ and $\beta_{12}=\beta_{1} \cdot\left(H \cdot K-\alpha_{1}\right)$. Then $H \cdot K=\beta_{11}+\beta_{12}+\sum_{2}^{n} \beta_{i}$ is a decomposition into $n+1$ closed sets between each pair of which both $H$ and $K$ are irreducible. We then have the contradiction that $Z-F$ has at least $n+1$ principal components.

7. THEOREM. For the bounded decomposable continuum $F$ to be the frontier of an infinity of components of its complement, it is necessary and sufficient that $F$ be the union of two continua $H$ and $K$ such that for every integer $n$ the set $H \cdot K$ is the sum of $n$ closed sets between each pair of which $H$ and $K$ are irreducible.

Proof. The necessity of these conditions was shown in

$†$ See reference to paper by the author under $\$ 1$. 
the proof of the theorem referred to in $\S 6$. That they are sufficient follows from $\$ 5$, Corollary.

8. Two Examples. It might be inferred that the statements of the theorems in $\$ \$ 6$ and 7 are unnecessarily complicated and that, if $H \cdot K$ is the sum of an infinite set of closed sets between each pair of which both $H$ and $K$ are irreducible, then $Z-F$ has an infinite set of principal components. This is not in general true. The following examples show the existence of two continua $H$ and $K$ having these properties, but such that $Z-F$ has no principal component in Ex. I and but two principal components in Ex. II.

EXAMPLE I. Let $Q=O A B C$ be a closed rectangle, such that the lengths of $O A$ and $A B$ are 1 and $1 / 2$ unit, respectively. Let $q$ be the frontier of $Q$. Let $M$ be a Cantor set extending from $O$ to $A$, whose complementary open intervals $\left\{I_{n}\right\}$ are ordered according to size.

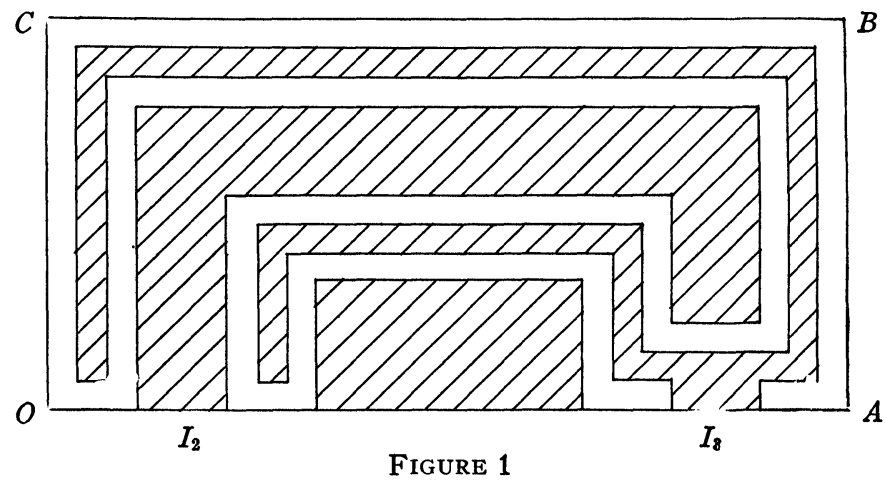

Now let a finite ordered set of closed rectangles, each of width $d$ and of length greater than $d$, such that each rectangle has in common with the one preceding and the one following two different squares of side $d$ but no point in common with any other rectangle, be called a band $\dagger$ of width $d$. It is

$\dagger$ For a complete discussion of the "method of bands" as applied to indecomposable continua see B. Knaster, Un continu dont tout sous-continu est indécomposable, Fundamenta Mathematicae, vol. 3, pp. 247-287. See also a memoir by K. Yoneyama, Tôhoku Mathematical Journal, vol. 12, pp. 60-62. 
easily seen that there is in $Q$ a unique band $B_{1}$ of width $1 / 3$, which is contiguous to all points of $q-I_{1}$ and whose frontier $q_{1}$ is the union of $q-I_{1}$ and a broken line $b_{1}$ meeting $q$ only in the end-points of $I_{1}$. Then $Q-B_{1}$ consists of $I_{1}$ and a simply connected region $E_{1}$ whose frontier is $I_{1}+b_{1}$; let $G_{1}=Q-B_{1}$.

Likewise, in $B_{1}$ there is a unique band $B_{2}$ of width $1 / 3^{2}$ whose frontier $q_{2}$ is the union of $q_{1}-I_{1}$ and a broken line $b_{2}$ meeting $q_{1}$ only in the end-points of $I_{2}$. Then $G_{2}=B_{1}-B_{2}$ consists of $I_{2}$ and a region $E_{2}$ whose frontier is $I_{2}+b_{2}$. This construction can be repeated indefinitely; in the figure the shaded area is $G_{1}+G_{2}+G_{3}$; the unshaded with its border is $B_{3}$.

Set $H=Q-\sum_{1}^{\infty} G_{n}=\prod_{1}^{\infty} B_{n}$. Obviously $H$ is a continuum. The Cantor set $M$ may be regarded as the sum of an infinity of closed sets without common points. These are the set $O+A$, an enumerable set of sets each consisting of the endpoints of an interval $I_{n}$, and a non-enumerable set of sets each of which is one of the other points of $M$. Let $\alpha$ be any of these sets. It is comparatively easy to show that $H$ is irreducible between each pair of sets $\alpha$.

Let $K$ be the continuum symmetrical to $H$ with respect to $O A$. Then $K$ is also irreducible between each pair of sets $\alpha$ and $H \cdot K=\sum \alpha$. But each component of $Z-F$ has for its frontier a pair of continua of condensation of $H$ and $K$.

ExAmple II. This is a variation of Ex. I. Take a closed rectangle $Q$ whose length and width are $43 / 27$ units and 1 unit respectively. Let $a b$ be one side and let the points $c, d, e$ and $f$ divide $a b$ into five intervals of lengths $a c=1 / 3$; $c d=2 / 27 ; d e=1 ; e f=2 / 27 ; f b=1 / 9$. Let $\left\{I_{k}\right\}$ be an enumerable set of open intervals divided into three classes as follows: $\left\{I_{3 n}\right\}$ is the set of complementary intervals of a Cantor set $M$ in the interval de ordered as in Ex. I; $\left\{I_{3 n-2}\right\}$ and $\left\{I_{3 n-1}\right\}$ are two other sets, of which the first are $I_{1}=c d$ and $I_{2}=e f$, and the others will be defined later. Let $q$ be the frontier of $Q$.

For $k=1,2,3$, let $B_{k}, q_{k}, b_{k}, E_{k}$, and $G_{k}$ be defined as in Ex. I. For $n=2$, let $I_{k}=I_{3 n-2}=I_{4}$ be an open interval of length $1 / 9$ at the center of one of the longest segments of 
$b_{1}$ perpendicular to, but not meeting, $a b$. Then $B_{4}$ is a band of width $1 / 3^{4}$ contiguous to $q_{3}-I_{4}$. This gives $q_{4} b_{4}, E_{4}$, and $G_{4}$. In this case, we note that $E_{1}+G_{4}$ is a simply connected region whose frontier is $I_{1}+\left(b_{1}-I_{4}\right)+b_{4}$. In like manner, we let $I_{5}$ be an open interval of length $1 / 9$ at the center of one of the longest segments of $b_{2}$ perpendicular to and not meeting $a b$ and define $B_{5}, q_{5}, b_{5}, E_{5}$, and $G_{5}$. Here $E_{2}+G_{5}$ is a simply connected region whose frontier is $I_{2}+\left(b_{2}-I_{5}\right)+b_{5}$.

We now return to $I_{6}$, which has been defined above. Let this process be repeated indefinitely. Each $I_{3 n}$ lies on $d e$; each $I_{3 n-2}$ is an open interval of length $1 / 9$ at the center of one of the longest segments of $b_{3 n-5}$ perpendicular to and not meeting $a b$; and similarly for $I_{3 n-1}$. In the figure the shaded

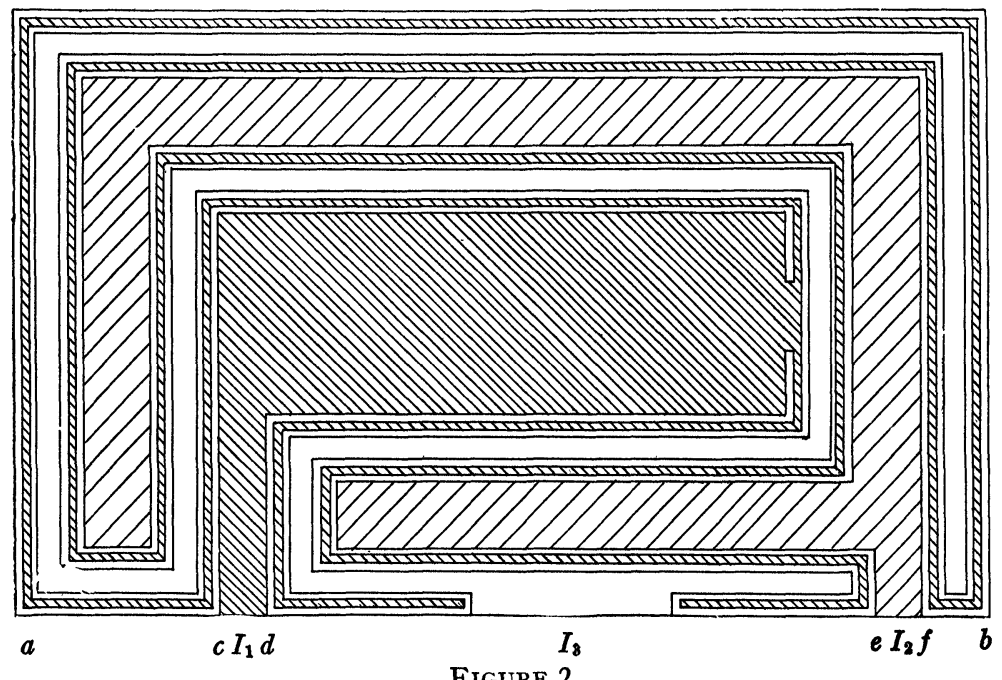

FIGURE 2

areas are $E_{1}+G_{4}$ and $E_{2}$; the narrower white area is $B_{4}$; and the other white area is $E_{3}$. For every value of $k, Q$ is the union of $B_{k}$ and a finite number of the sets $G_{k}$. No two of the sets $G_{3 n}$ or of the corresponding regions $E_{3 n}$ have common points. For each $n, E_{1}+G_{4}+\cdots+G_{3 n-2}$ and $E_{2}+G_{5}$ $+\cdots+G_{3 n-1}$ are simply connected regions whose frontiers 
are the union of $I_{1}$ and $I_{2}$, respectively, with a broken line interior to $Q$.

Let $H=Q-\sum_{1}^{\infty} G_{k}=\prod_{1}^{\infty} B_{k}$. Obviously $H$ is a continuum. The set $a c+f b+M$ is a closed set which may be regarded as the sum of an infinity of closed sets without common points. These are the set $a c+f b$, an enumerable set of sets each consisting of the end-points of an interval $I_{3 n}$, and a non-enumerable set of sets each of which is one of the other points of $M$. Let $\alpha$ denote any of these sets. It is comparatively easy to show that $H$ is irreducible between each pair of $\operatorname{sets} \alpha$.

Reflect the above figure about $a b$ and denote corresponding sets by primes. Then $K=H^{\prime}$ is a continuum irreducible between each pair of sets $\alpha$ and $H \cdot K=\sum \alpha$. The components of $Z-F$, where $F=H+K$, are the exterior of the rectangle $Q+Q^{\prime}$, the enumerable set of regions $G_{3 n}+G_{3 n}^{\prime}$, each of which has as its frontier a pair of continua of condensation of $F$, and two other regions. These are $\sum_{1}^{\infty} G_{3 n-2}$ $+\sum_{1}^{\infty} G_{3 n-2}^{\prime}$ and $\sum_{1}^{\infty} G_{3 n-1}+\sum_{1}^{\infty} G_{3 n-1}^{\prime}$. Each of these has the frontier $F$ and is therefore a principal component of $Z-F$.

The essential difference between the two examples is that in Ex. I, $H \cdot K$ cannot be divided into any finite number of closed sets between each pair of which $H$ and $K$ are irreducible, while in Ex. II, $H \cdot K$ can be divided in to precisely two, but no more, such sets, namely the sets $a c+f b$ and $M$. Thus Ex. II is in strict accordance with $\$ \S 6$ and 7. 